\title{
Analysis of Galileo signal-in-space range error and positioning performance during 2015-2018
}

Weiwang Wu' ${ }^{1}$ Fei Guo ${ }^{1 *}$ and Jiazhu Zheng ${ }^{2}$

\begin{abstract}
A long-term analysis of signal-in-space range error (SISRE) is presented for all healthy Galileo satellites, and the first pair of full operational capability satellites in wrong elliptical orbits. Both orbit and clock errors for Galileo show an obvious convergence trend over time. The annual statistical analyses show that the average root mean squares (RMSs) of SISRE for the Galileo constellation are $0.58 \mathrm{~m}$ (2015), $0.29 \mathrm{~m}$ (2016), $0.23 \mathrm{~m}$ (2017), and $0.22 \mathrm{~m}$ (2018). Currently, the accuracy of the Galileo signal-in-space is superior to that of the global positioning system (GPS) Block IIF ( $0.35 \mathrm{~m}$ ). In addition, the orbit error accounts for the majority of Galileo SISRE, while the clock error accounts for approximately one-third of SISRE due to the high stability of the onboard atomic clock. Single point positioning results show that Galileo achieves an accuracy of 2-3 m, which is comparable to that of GPS despite the smaller number of satellites and worse geometry. Interestingly, the vertical accuracy of Galileo, which uses the NeQuick ionospheric model, is higher than that of GPS. Positioning with single frequency E1 and E5 show a higher precision than E5a and E5b signals. Regarding precise point positioning (PPP), the results indicate that a comparable positioning accuracy can be achieved among different stations with the current Galileo constellation. For static PPP, the RMS values of Galileo-only solutions are within $1 \mathrm{~cm}$ horizontally, and the vertical RMSs are mostly within $2 \mathrm{~cm}$ horizontally. For kinematic PPP, the RMSs of Galileo-only solutions are mostly within $4 \mathrm{~cm}$ horizontally and $6 \mathrm{~cm}$ vertically.
\end{abstract}

Keywords: Galileo, Signal in space range error (SISRE), Single point positioning (SPP), Precise point positioning (PPP)

\section{Introduction}

Galileo, the European global navigation satellite system (GNSS), has achieved substantial progress in recent years. Four in-orbit validation (IOV) satellites were launched in 2011 and 2012. After successful completion of the IOV phase, the Galileo program is moving into the full operational capability (FOC) phase. The first pair of Galileo FOC satellites, FOC-1 and 2, was launched in August 2014. Unfortunately, an unexpected injection failure was reported, and the vehicle were sent into an incorrect orbit. Thereafter, a new type of dispenser (Ariane 5) has been designed to carry four FOC satellites into

\footnotetext{
*Correspondence: fguo@whu.edu.cn

1 School of Geodesy and Geomatics, Wuhan University, 129 Luoyu Road, Wuhan 430079, China

Full list of author information is available at the end of the article
}

orbit at once in subsequent launches, which enabled a substantial increase in the number of satellites in orbit. Currently, the Galileo constellation comprises 4 IOV satellites and 22 FOC satellites transmitting on five frequencies, i.e., E1, E5a, E5b, E5, and E6. Following the declaration of initial services on December 15, 2016, Galileo has been able to independently provide positioning, navigation, and timing services for users around the world.

A number of researchers have investigated the performance of the earlier Galileo satellites. An analysis of receiver-specific measurement errors for IOV and FOC satellites on all Galileo signals was discussed by Zaminpardaz and Teunissen [1]. As an accuracy indicator of a broadcast satellite orbit and clock offset, signal-in-space range error (SISRE) is driven by the space segment characteristics and ground control segment capabilities [2]. SISRE contributes as one of the key metrics commonly 
employed in GNSS performance monitoring. Previous research was primarily dedicated to the SISRE characteristics of the legacy global positioning system (GPS) and global navigation satellite system (GLONASS) [3-5]. For the new emerging BeiDou navigation satellite system (BDS), Chen et al. [6] and Wu et al. [7] analyzed the longterm behaviors of the orbit, clock and SISRE for the three different types of orbits, i.e., geostationary Earth orbit (GEO), inclined geosynchronous satellite orbit (IGSO) and medium Earth orbit (MEO) satellites. In the initial SISRE assessment of Galileo in the phase of IOV Lucas Rodriguez [8] and Montenbruck et al. [2] reported an initial accuracy of 1-2 m. Perea et al. [9] analyzed the error distribution of the clock and orbit as well as the SISRE with 4 months of Galileo data in 2015. They discovered that the behavior of Galileo SISRE is primarily driven by satellite clock error. Montenbruck et al. [10] reviewed the fundamental concepts and underlying assumptions of SISRE and assessed the multi-GNSS SISRE throughout 2017, and reported a representative global average rootmean-square (RMS) SISRE of $0.2 \mathrm{~m}$ for Galileo.

Being compatible and interoperable with GPS, Galileo is often evaluated along with combination of GPS/Galileo from the early days of the Galileo system test bed [1115]. To investigate the potential contribution of the first pair of Galileo FOC satellites that were sent into incorrect, highly eccentric orbits, Paziewski et al. [16] utilized the FOC-1 and 2 satellites in precise relative positioning, and observed no negative effect, which means that the two satellites are applicable for most geodetic solutions. This claim, however, holds true only if multi-GNSS experiment (MGEX) employed precise products rather than the broadcast ephemeris.

Given that the Galileo constellation has undergone extensive deployment in recent years, the previous results (of a few satellites) may not be representative of the current or future performance of the full constellation. In this research, we analyze the long-term behavior of the Galileo broadcast ephemeris from 2015 through 2018 for all healthy satellites and the incorrect orbit satellites FOC-1 and 2. In addition, the performances of standard point positioning (SPP) and precise point positioning (PPP) are evaluated.

\section{Methods}

SISRE is defined as the difference between the true satellite position and time and those broadcast by a navigation message. In this concept, for each satellite, an individual SISRE comprises the vector of orbit errors $d_{\text {orb }}=(d A, d C, d R)$ in the along-track $(d A)$, cross-track $(d C)$ and radial $(d R)$ directions coupled with the clock errors $d t$ at an arbitrary epoch. For statistics, the global average SISRE is computed as the RMS of the instantaneous SISRE for all satellites and epochs. Accordingly, the global average SISRE is an RMS statistic of the orbit and clock errors that are projected in the signal propagation direction (line-of-sight to the users), which can be simply expressed as

$$
S I S R E=\sqrt{\left(W_{R} \cdot d R-d T\right)^{2}+W_{A, C}^{2} \cdot\left(d A^{2}+d C(1)\right)}
$$

where $W_{R}$ and $W_{A, C}$ are the constellation-specific weighted factors (or projection factors) for the radial components and along/cross-track components, respectively.

To evaluate the orbit errors in the total positioning error budget, the orbit-only contribution to the SISRE is expressed as follows:

$$
\operatorname{SISRE}_{\text {orbit }}=\sqrt{W_{R}^{2} \cdot d R^{2}+W_{A, C}^{2} \cdot\left(d A^{2}+d C^{2}\right)}
$$

Note that $W_{R}$ and $W_{A, C}$ are the satellite-specific value and epoch-specific value due to the time-varying geometry. For simplicity, we use the constant $W_{R}$ of 0.98 for both constellations, while $W_{A, C}^{2}$ is set to 0.016 and 0.020 for the Galileo constellation and GPS constellation, respectively [2].

The precise orbit and clock products with an accuracy of $2-3 \mathrm{~cm}$ provided by the International GNSS Service (IGS) [17] can be used as a reference for the broadcast ephemeris error statistics. However, the compatibility and consistency of the orbit and clock information should be taken into account when the comparing broadcast ephemeris and precise products.

Note that all precise orbit products provided by the IGS are referred to the center of mass (CoM) of the spacecraft, while the broadcast orbits of the Galileo and GPS are referred to the antenna phase center (APC) [18]. Consequently, the known phase center offset (PCO) for every satellite should be utilized for the correction of CoM to APC. In this study, the phase center locations for the E1 and E5a frequencies as provided in GSA [19] has been employed for this purpose. For the newly available launch-9 satellites, which do not have access to their PCO, the average PCO of nominal FOC satellites is applied for these four satellites.

Conventionally, the MGEX precise products provide the clock offset of Galileo based on the signal combination E5a/E1, while the clock references differ in two types of Galileo navigation messages. The first type of navigation message is the freely accessible navigation (FNAV) message, which is referred to the signal combination $\mathrm{E} 5 \mathrm{a} / \mathrm{E} 1$, and the second type of navigation message is the integrity navigation (INAV) message, which is referred 
to the combination E5b/E1. To be consistent with that of precise clock products, the MGEX differential code bias (DCB) estimates can be used to convert the clock offset of the INAV message $\Delta t_{I N A V}$ to the datum E5a/E1 as

$$
\begin{aligned}
\Delta t_{I F}(E 1, E 5 a)= & \Delta t_{I N A V}(E 1, E 5 b) \\
& +\frac{f_{E 5 a}^{2}}{f_{E 1}^{2}-f_{E 5 a}^{2}} D C B_{C 1 X C 5 X}^{s a t} \\
& -\frac{f_{E 5 b}^{2}}{f_{E 1}^{2}-f_{E 5 b}^{2}} D C B_{C 1 X C 7 X}^{s a t}
\end{aligned}
$$

where $\Delta t_{I F}(E 1, E 5 a)$ is the clock offset of the precise product generated from the observation of the ionosphere-free (IF) combination E5a/E1; $f_{\mathrm{E} 1}, f_{\mathrm{E} 5 \mathrm{a}}$, and $f_{\mathrm{E} 5 \mathrm{~b}}$ are the carrier frequencies of signals E1, E5a, and E5b, respectively, and the code observations correspond to C1X, C5X, and C7X, respectively.

Similar with the time group delay (TGD) parameter in the navigation message of GPS and BDS, the broadcast group delay (BGD) is one of the Galileo broadcast parameters that is commonly employed to compensate the inter-frequency biases of satellites for single-frequency users. Every Galileo satellite broadcasts unique BGD offsets, i.e., $B G D(E 1, E 5 a)$ and $B G D(E 1, E 5 b)$, and they are contained on FNAV and INAV messages according to the Galileo interface control document (ICD) [20]. The direct relationships between BGD parameters and $\mathrm{DCB}$ estimates are expressed as follows:

$$
\left\{\begin{array}{l}
B G D(E 1, E 5 a)=-\frac{f_{E 5 a}^{2}}{f_{E 1}^{2}-f_{E 5 a}^{2}} D C B_{C 1 X C 5 X}^{s a t} \\
B G D(E 1, E 5 b)=-\frac{f_{E 5 b}^{2}}{f_{E 1}^{2}-f_{E 5 b}^{2}} D C B_{C 1 X C 7 X}^{s a t}
\end{array}\right.
$$

To investigate the quality of Galileo BGD parameters, DCB estimates with an accuracy of 0.1 ns provided by the German Aerospace Center (DLR) were applied as reference values. Figure 1 shows the statistics of the differences between BGDs and DCBs for each Galileo satellite from 2015 to 2018. The BGD parameters for the entire constellation show agreement with the DCB estimates.

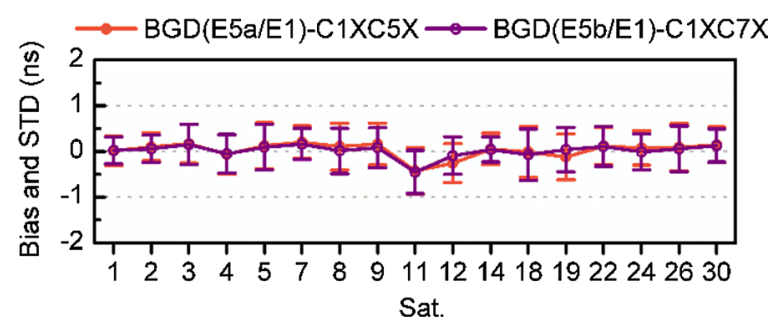

Fig. 1 Statistics of the differences between BGDs and DCBs for Galileo satellites (E1-E30) from 2015-2018. Dots and bars indicate the mean values and the STDs, respectively, of the daily biases differences
The mean biases of their differences are near zero and the standard deviation (STD) is $\pm 0.5 \mathrm{~ns}$, which support the relationship between BGDs and DCBs, as shown in Eq. 4. The older IOV E11 shows the exception that the mean biases are 0.5 ns below zero, which can be attributed to the actual BGD that has changed from the ground calibration value over time.

Consequently, for single frequency positioning, the Galileo satellites clock $\Delta t_{S V}$ can be corrected by BGD parameters or the more accurate DCB estimates with Eq. 5.

$$
\left\{\begin{array}{l}
\Delta t_{S V}(E 1)=\Delta t_{I N A V}(E 1, E 5 b)-B G D(E 1, E 5 b) \\
\Delta t_{S V}(E 5 b)=\Delta t_{I N A V}(E 1, E 5 b)-\left(\frac{f_{E 1}}{f_{E E b}}\right)^{2} B G D(E 1, E 5 b) \\
\Delta t_{S V}(E 5 a)=\Delta t_{F N A V}(E 1, E 5 a)-\left(\frac{f_{E 1}}{f_{E 5 a}}\right)^{2} B G D(E 1, E 5 a)
\end{array}\right.
$$

Due to the lack of definition of BGD correction for E5 observation in Galileo ICD, we utilize the DCB estimates instead for $\Delta t_{S V}(E 5)$ compensation, that is,

$$
\begin{aligned}
\Delta t_{S V}(E 5)= & \Delta t_{F N A V}(E 1, E 5 a) \\
& +\frac{f_{E 5 a}^{2}}{f_{E 1}^{2}-f_{E 5 a}^{2}} D C B_{C 1 X C 5 X}^{s a t} \\
& +D C B_{C 1 X C 8 X}^{s a t} .
\end{aligned}
$$

where C8X refers to the code observation of the E5 signal.

\section{Datasets and processing}

A time span of more than 3 years (April 2015-July 2018) of ephemeris data were used for SISRE computations and its evolution analysis. The broadcast ephemeris originates from the merging broadcast ephemeris file (BRDM), which is routinely generated as part of MGEX. The BRDM file covers not only the legacy GPS and GLONASS but also the new emerging Galileo, BDS, and QZSS constellations. The multi-constellation orbit and clock products of GeoForschungsZentrum (GFZ) with an accuracy of a few centimeters were adopted as precise references. An outlier screening was applied to remove the extremely large errors. Given a time series of SISRE, the values that deviate from the mean by more than three times the STDs will be regarded as outliers and excluded from the statistics.

To further verify the quality of a signal in space, positioning tests were performed. Data sets of globally distributed MGEX stations (Fig. 2) for the day of year (DOY) 151-157, 298-304, and 200-206 in 2016, 2017, and 2018, respectively, were employed for SPP and PPP solutions. For the current Galileo constellation, most MGEX stations are able to simultaneously track 4-7 satellites in a few hours. Therefore, 3-h observations with a similar number of visible Galileo or GPS satellites were 


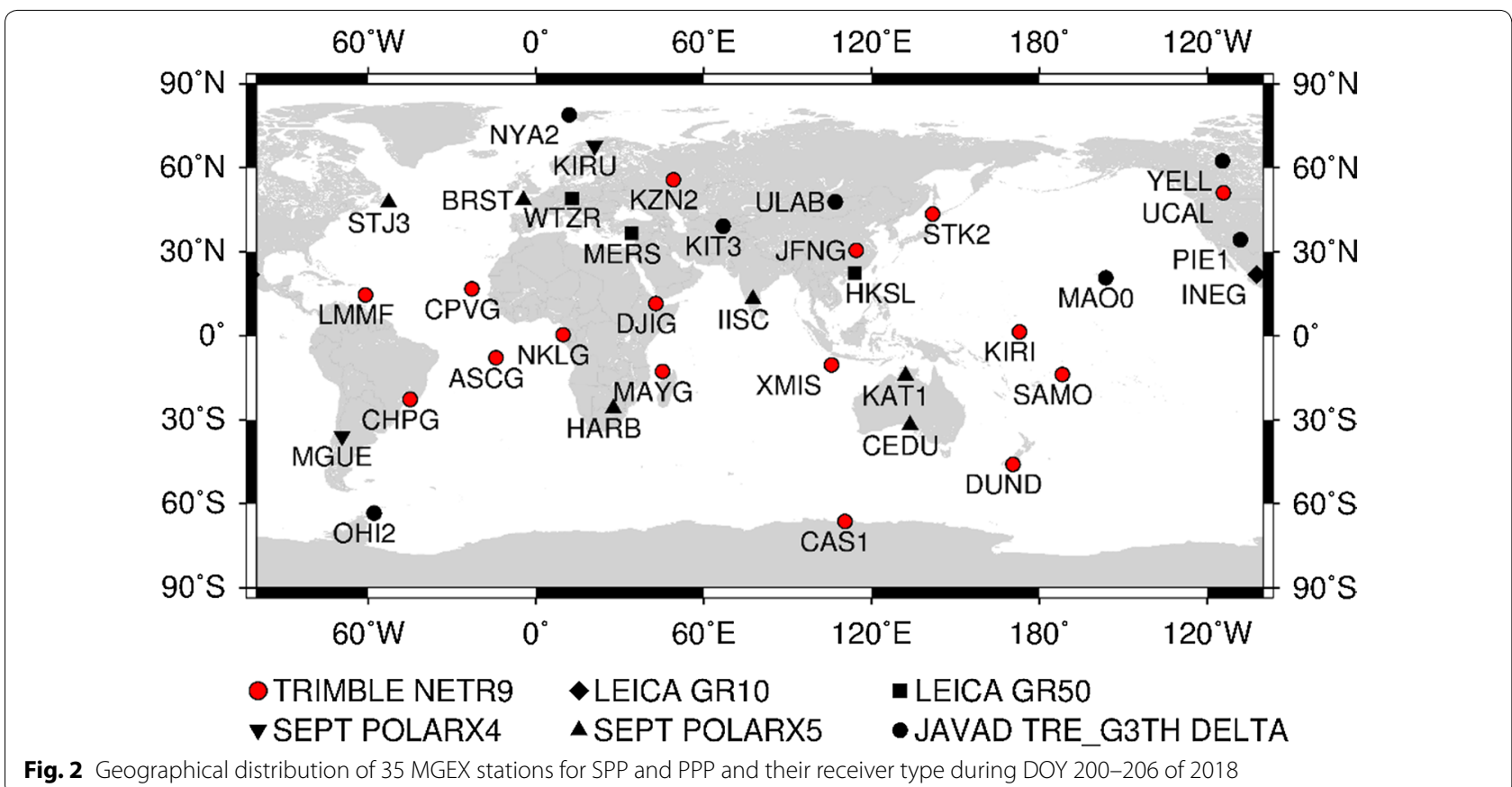

Table 1 Processing strategies for SPP and PPP

\begin{tabular}{|c|c|c|}
\hline Items & SPP & PPP \\
\hline Observation & $\begin{array}{l}\text { Galileo: E1, E5a, E5b, E5 } \\
\text { GPS: L1 }\end{array}$ & $\begin{array}{l}\text { Galileo: E1/E5a } \\
\text { GPS: L1/L2 }\end{array}$ \\
\hline Sampling rate & \multicolumn{2}{|l|}{$30 \mathrm{~s}$} \\
\hline Elevation cutoff & \multicolumn{2}{|l|}{$10^{\circ}$} \\
\hline Estimation method & Weighted least squares & Kalman filter \\
\hline Weight scheme & $\begin{array}{l}\text { Elevation-dependent weight; } 0.3 \mathrm{~m} \\
\text { for raw code }\end{array}$ & Elevation-dependent weight; $3 \mathrm{~mm}$ for raw phase \\
\hline Ionospheric delay & $\begin{array}{l}\text { Galileo: NeQuick model } \\
\text { GPS: Klobuchar model }\end{array}$ & Eliminated by IF combination \\
\hline Tropospheric delay & Not applied & $\begin{array}{l}\text { Dry component: GPT model; Wet component: estimated as random walk process; } \\
\text { Global Mapping Function [21] }\end{array}$ \\
\hline Timing group delay & $\mathrm{BGD} / \mathrm{TGD}$ correction & Not applied \\
\hline Satellite PCO & Not applied & Galileo: MGEX Conventional values and igs08.atx since DOY 269, 2016; GPS: igs08.atx \\
\hline Receiver PCO & Not applied & Corrected PCO and PCV \\
\hline Phase-windup effect & Not applied & Corrected [7] \\
\hline Receiver clock & \multicolumn{2}{|l|}{ Estimated, white noise process } \\
\hline Inter system bias & \multicolumn{2}{|c|}{ Estimated, constant over time (Galileo/GPS combined only) } \\
\hline Station coordinate & Estimated, white noise process & $\begin{array}{l}\text { Estimated, constants for static PPP; white noise process for kinematic PPP, variance } \\
\text { of position is set to } 100^{2} \mathrm{~m}^{2}\end{array}$ \\
\hline
\end{tabular}

employed for comparison. Note that a positioning test was not performed in 2015 due to the lack of sufficient visible satellites. The processing strategies for SPP and PPP are summarized in Table 1 . The positioning results were compared with the daily or weekly solutions from the SINEX file. The reference coordinates have an accuracy of a few millimeters.

\section{SISRE evolution analysis}

The broadcast orbit and clock error for Galileo and the GPS IIF satellites equipped with stable rubidium atomic frequency standards (RAFS) are presented. The statistics of SISRE of the two constellations are presented and analyzed in this section. 


\section{Orbit error}

Figure 3 presents the time series of Galileo orbit errors in the along-track, cross-track, and radial components and the frequency histogram for the entire period. The orbit errors in the along- and cross-track directions are within $\pm 4.0 \mathrm{~m}$ in 2015 , whereas the orbit errors in the same components decreased to $\pm 2.0 \mathrm{~m}$ in 2016 and gradually stabilized at $1-2 \mathrm{~m}$ in the following two years. For individual IOV and FOC satellites, the mean biases are within a few centimeters, and the differences in the STDs between IOVs and FOCs are marginal.

Figure 4 shows the corresponding orbit errors of the GPS IIF satellites. Different from Galileo, the GPS orbit errors behave as stable random noises, and no significant difference among the 3 years was observed. The STDs of the broadcast GPS orbit error range were $77-81 \mathrm{~cm}$ in the along-track, $72-77 \mathrm{~cm}$ in the cross-track, and $18-19 \mathrm{~cm}$ in the radial components. Compared with Fig. 3, Galileo has a comparable orbit precision with the GPS in the radial direction, while the orbit in the alongand cross-track directions distinctly outperform that of the GPS. Note that the histogram of the radial component error has a minor skew and the mean values are approximately $5 \mathrm{~cm}$, which may be attributed to the PCO with an uncertainty of a few centimeters.
In addition to the general aspects, some anomalies are observed in Figs. 3 and 4. Note that Galileo orbit errors show distinct jumps in September 2016, which was reported as system testing for all operational satellites in a notice advisory to Galileo users (NAGU). Some unexpected large errors frequently and synchronously occurred in the three directions. The Age of Data (AOD) for these periods was considerably larger than that of the other periods. As a newly launched satellite, the orbit errors of GPS pseudo-random noise (PRN) 32 are larger at the early stage, particularly in the radial component, and gradually normalize after a short period of on-orbit testing.

Two incorrect FOC satellites (E14/E18) have started broadcasting ephemeris data since August 2016 after a series of orbit maneuvers and adaptions made by a ground mission segment (GMS) [22]. However, E14 and E18 are not part of the operational constellation, and the broadcast ephemeris was flagged as "unhealthy." The top panel of Fig. 5 shows a three-day arc of the broadcast ephemeris errors of E14. Some large orbit errors periodically occurred, which is highly correlated with the orbit altitude. To provide a better perspective, the scatters of E14/E18 orbit errors and clock bias versus the orbit latitude based on one-month data in June 2018 are

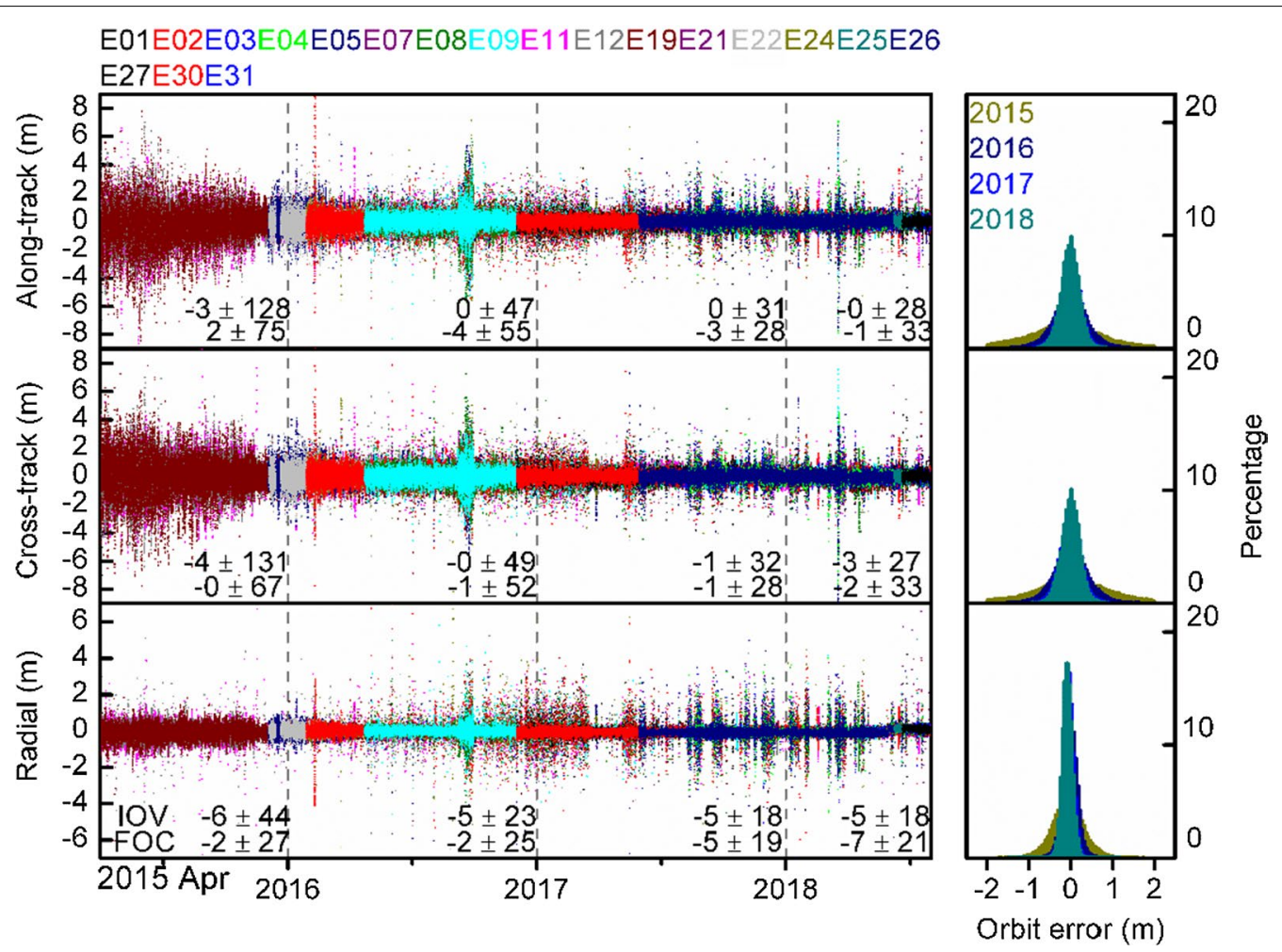

Fig. 3 Time series of Galileo FNAV broadcast orbit errors (left) and the frequency histogram (right). Mean and STDs of IOV and FOC satellites for each year are indicated at the bottom in centimeters 


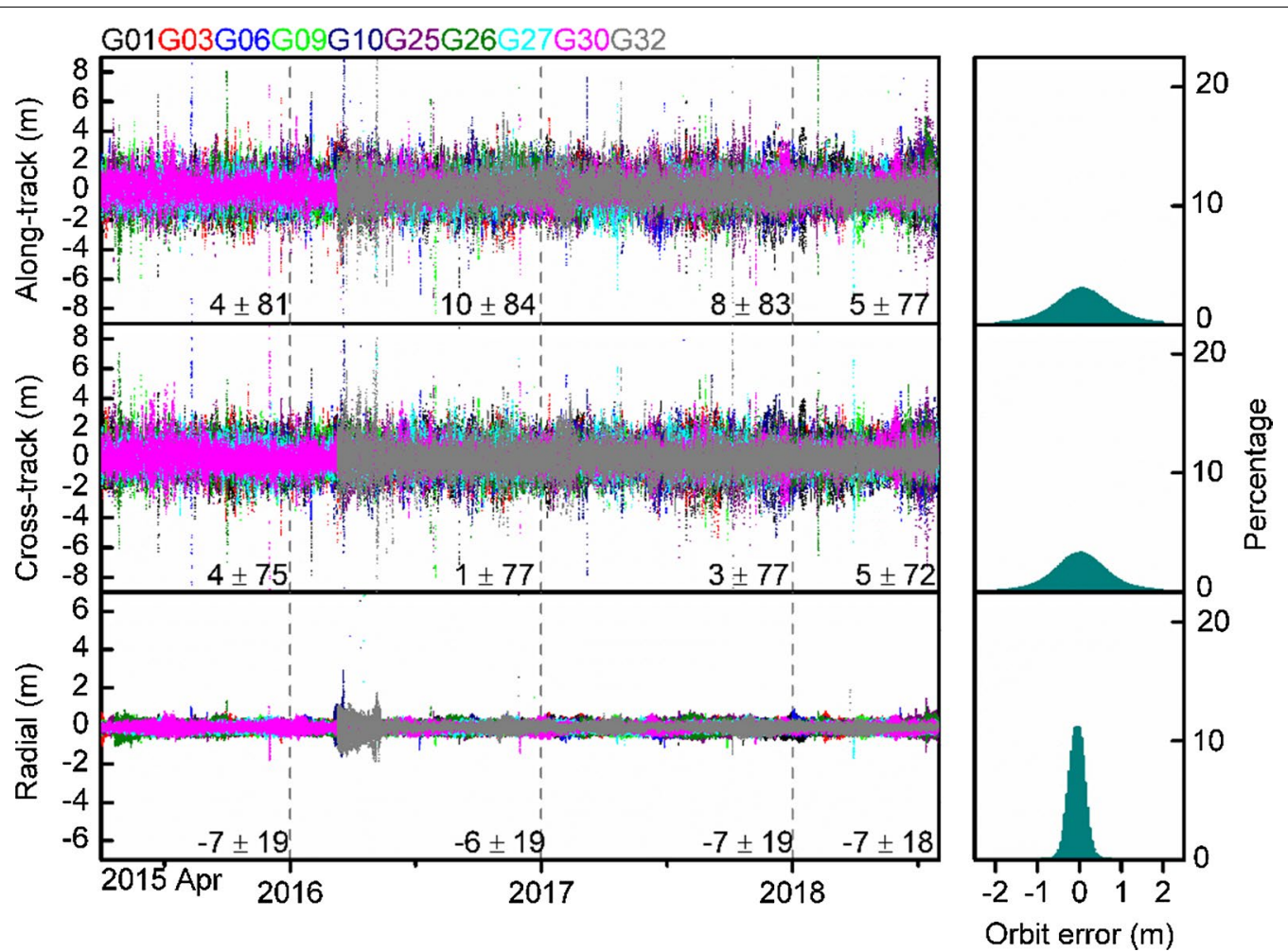

Fig. 4 Time series of GPS IIF LNAV broadcast orbit errors (left) and the frequency histogram (right). Mean and STDs for each year are indicated at the bottom in centimeters

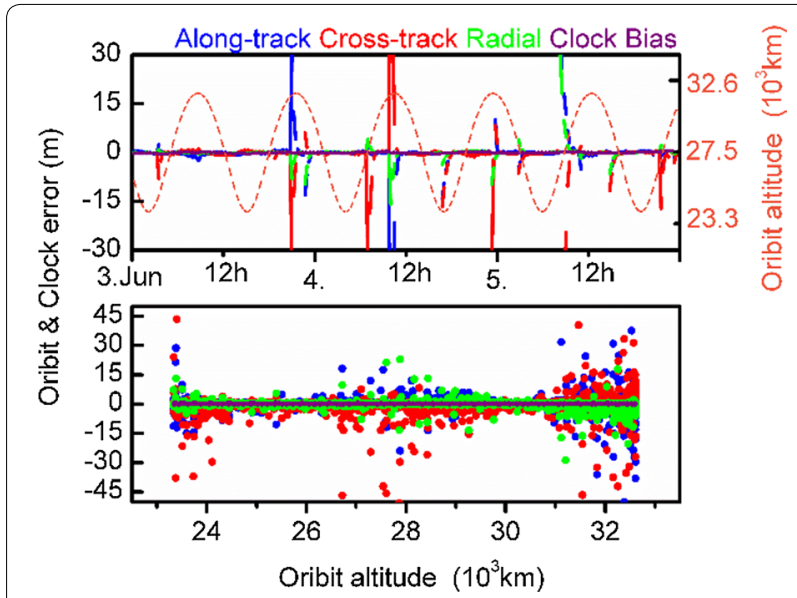

Fig. 5 Time series of orbit errors, clock bias and orbit altitude (top) of Galileo E14 (June 3-5 2018) and orbit errors and clock bias of E14/E18 versus orbit altitude (bottom) in June 2018

presented in Fig. 5 (bottom). When the satellite passes through the perigee, apogee or the intermediate point, the orbit errors in the three components reach a maximum of tens of meters with a few exceptions, while the clock biases are not affected and remain within a small range of values. The hardly predictable satellite attitude of the elliptical orbit with respect to the MEO is responsible for the anomaly near the perigee, apogee and intermediate point. The large orbit errors may be attributed to the imperfect attitude models of elliptical orbit determination for the broadcast ephemeris, which requires further investigation.

\section{Clock error}

Figure 6 shows the time series of clock errors and the frequency histogram for the same period. Likewise, the annual statistics of all Galileo IOV satellites and FOC satellites, as well as GPS IIF satellites are marked in the figure. The clock error decreases from approximately $60 \mathrm{~cm}$ in 2015 to approximately $25 \mathrm{~cm}$ in 2018 for the IOV and FOC satellites. For the GPS IIF satellites, with the exception of PRN 32, the clock errors run smoothly and are continuously maintained at a level of $30 \mathrm{~cm}$ in the entire period. The satellite-specific clock RMS is shown in Fig. 7. The RMS of the Galileo clock error decreases annually, which indicates an increasing accuracy of Galileo clock information. Note that the RMS values of E22 and E26 in 2016 are even larger than those in 2015 due to clock anomalies. With the exception of the newly E21 in 

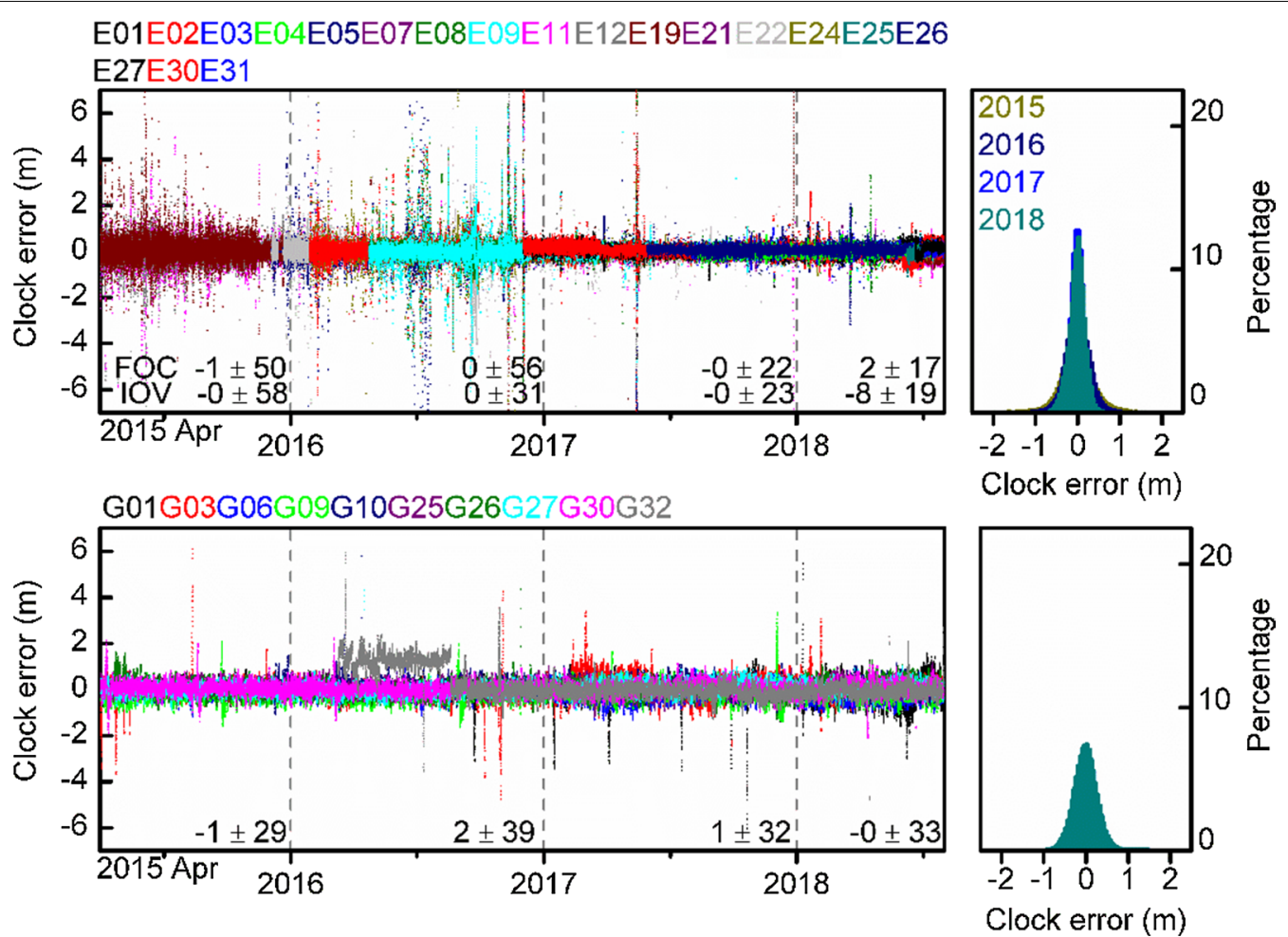

Fig. 6 Time series of Galileo FNAV (top) and GPS IIF LNAV (bottom) broadcast clock errors and frequency histogram (right). Mean and STDs for each year are indicated at the bottom in centimeters

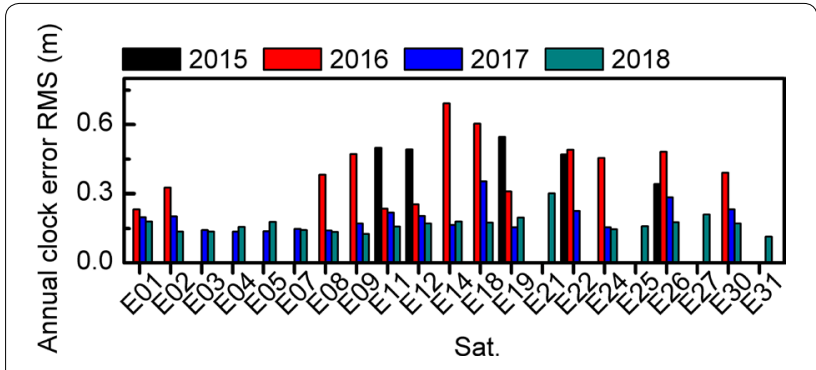

Fig. 7 Satellite-specific clock RMS errors for all Galileo satellites

its commissioning phase, all other Galileo satellites show clock RMS values between 0.11 and $0.21 \mathrm{~m}$ in 2018 .

Each Galileo satellite is equipped with four atomic clocks. Two of the clocks are RAFS clocks, such as those installed on GPS IIF satellites, and the other two clocks are passive hydrogen maser (PHM) clocks. To analyze the clock stability, the overlapping Allan deviation (ADEV) was used to graphically visualize the random characteristics of satellite clock behavior. The GFZ precise clock product is applied for ADEV computations. Figure 8 shows the median Allan deviations of ten 3-day arcs of Galileo and GPS IIF clock solutions for November 2017.

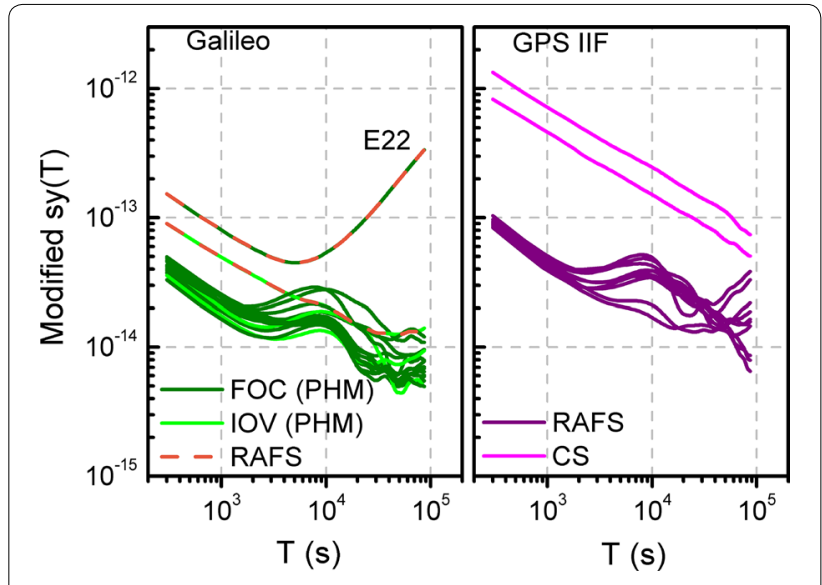

Fig. 8 Median ADEVs of Galileo (left) and GPS IIF (right) satellites

We observe that the performance of the IOV PHM clocks is similar to the performance of the FOC PHM clocks. The PHM clocks attain an ADEV of $1.2-4.3 \times 10^{-14}$ for integration times of 300 and $10,000 \mathrm{~s}$. This stability is twice that of the GPS IIF RAFS clocks, which further confirmed the excellent clock quality of Galileo. However, the two GPS cesium standard (CS) clocks are significantly worse than the GPS RAFS clocks by a factor of ten. Note 
that only two satellites-E11 and E22-operated with RAFS clocks during the analysis period and the ADEV of the satellite E11 RAFS shows the comparable stability with respect to the GPS IIF RAFS. The satellite E22 RAFS clocks substantially increase from 10,000 s, which shows a continuously rising curve. This abnormal phenomenon can be attributed to the performance degradation, which has been confirmed in the following months. On December 8, 2017, the E22 satellite was removed from active service for constellation management purposes.

\section{SISRE overview}

Figure 9 shows the daily RMS of SISRE for all operational Galileo satellites and the GPS IIF (RAFS) satellites for the same period. Since the scatters of orbit and clock errors show a distinct convergence trend over time, the SISRE naturally exhibits a similar feature of convergence. The evolution of SISRE can be revealed from annual statistics, i.e., the annual mean values and STDs of Galileo FOCs SISRE are $0.41 \pm 0.14 \mathrm{~m}, 0.30 \pm 0.35 \mathrm{~m}, 0.21 \pm 0.17 \mathrm{~m}$, and $0.21 \pm 0.13 \mathrm{~m}$ in 2015, 2016, 2017, and 2018, respectively. For GPS IIF satellites, the SISREs had a stable STD of $0.35 \mathrm{~m}$ in the past few years. A comparison of the annual STDs of SISRE between Galileo and the GPS indicated that the accuracy of Galileo signal in space is distinctly inferior to that of the GPS in 2015, while it is comparative to the GPS in 2016 and currently superior to that of the GPS.

To reveal the contribution (error budget) of orbit and clock errors to the SISRE, the annual mean RMS of orbitonly and total SISREs are presented in Fig. 10. For the operational Galileo satellites, the annual mean values of total SISREs are $0.40-0.60 \mathrm{~m}, 0.24-0.38 \mathrm{~m}, 0.18-0.24 \mathrm{~m}$,

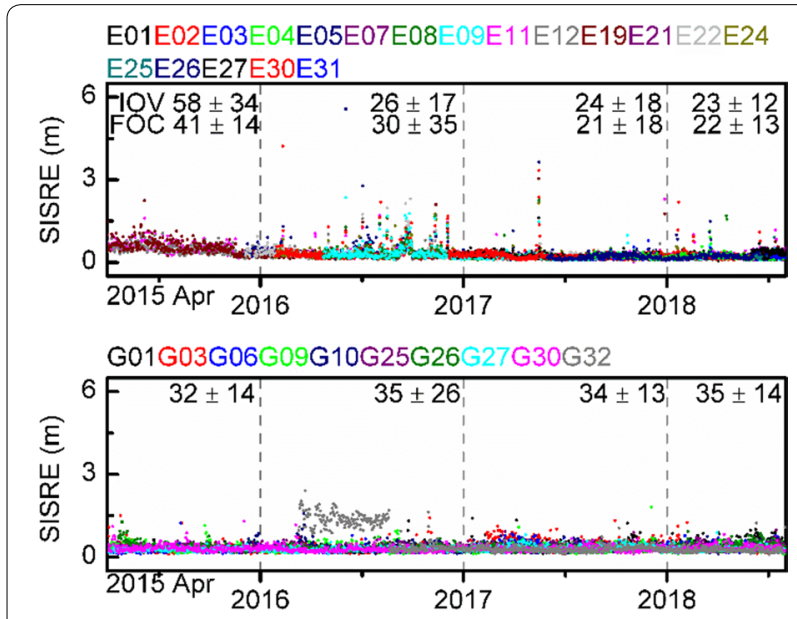

Fig. 9 Time series of Galileo FNAV (top) and GPS IIF LNAV (bottom) global average SISRE errors and annual statistics of mean and STD $(\mathrm{cm})$

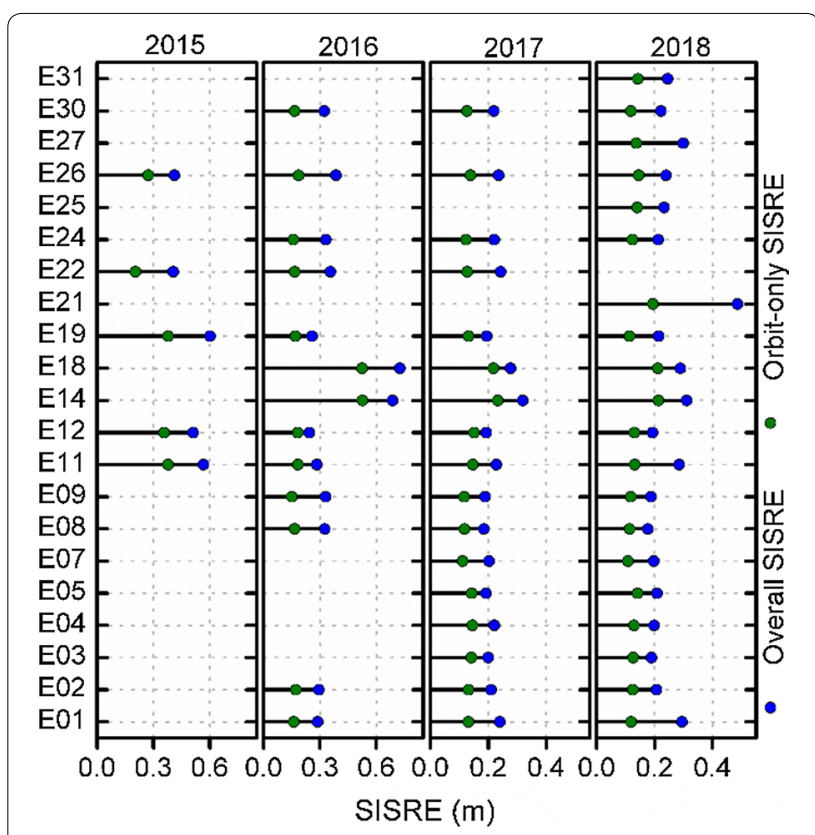

Fig. 10 Annual mean RMS of orbit-only (green dots) and global average (blue dots) SISREs for each Galileo satellite from 2015-2018

and $0.17-0.29 \mathrm{~m}$ in $2015,2016,2017$, and 2018, respectively. The orbit error accounts for the majority of Galileo SISRE, while the clock error accounts for approximately one-third of SISRE due to the high stability of the onboard atomic clock. Due to the large orbit errors (Fig. 5), the E14 and E18 show significantly larger SISREs than the other satellites in 2016 and 2017. The SISRE of the newly launched satellite E21 attains more than $0.50 \mathrm{~m}$ during its commissioning phase. Note that the Galileo GMS was updated from version 1.2 to 2.0.1 in March 2015, which included four additional sensor stations and an uplink station. The notable improvement in the space range accuracy of the Galileo signal can be attributed to the joint effects of the better coverage of sensor and uplink stations and the refinement of the clock and orbit determination on GMS [23].

The stable results for the period between January 2017 and July 2018 were used for the statistics of current broadcast ephemeris accuracy. Table 2 summarizes the individual orbit and clock error statistics, and the resulting SISRE values. The orbit errors of Galileo satellites that run in a nominal orbit are $0.249 \mathrm{~m}, 0.242 \mathrm{~m}$ and $0.174 \mathrm{~m}$ in the along-track component, cross-track component and radial component, while the orbit accuracy of FOC-1 and 2, which is run in the wrong elliptical orbit, is worse by a factor of 2-3. Different from orbit errors, the clock errors of two types of Galileo satellites are approximately $0.170 \mathrm{~m}$, which shows no significant difference. Consequently, the total SISREs are $0.227 \mathrm{~m}$ and $0.402 \mathrm{~m}$, and 
Table 2 Root mean square orbit errors in the along-track $(A)$, cross-track $(C)$, and radial $(R)$ direction and the clock errors $(T)$, total and orbit-only SISREs of Galileo and GPS IIF (RAFS) satellites (units: $m$ )

\begin{tabular}{|c|c|c|c|c|c|c|c|}
\hline System & Type & $A$ & $C$ & $R$ & $T$ & SISRE & SISRE (orb) \\
\hline Galileo & FNAV & & & & & & \\
\hline Nominal & & 0.249 & 0.242 & 0.174 & 0.171 & 0.227 & 0.135 \\
\hline FOC-1, 2 & & 0.712 & 0.738 & 0.335 & 0.169 & 0.402 & 0.330 \\
\hline GPS & LNAV & & & & & & \\
\hline$\| F$ & & 0.615 & 0.582 & 0.186 & 0.314 & 0.351 & 0.219 \\
\hline
\end{tabular}

the orbit-only SISREs are $0.135 \mathrm{~m}$ and $0.330 \mathrm{~m}$ for these two types of Galileo satellites.

\section{Positioning performance}

The SPP and PPP results of a few representative stations are presented and analyzed in this section. For comparison, both SPP and PPP tests were individually performed with Galileo-only, GPS-only, and Galileo/GPS combined observations.

\section{SPP results and analysis}

We first evaluated the positioning performance of Galileo with E1 frequency. Figure 11 shows the statistics of the positioning errors, number of satellites, and PDOPs of the three solutions. Note that L1 frequency observations were employed for the GPS solutions based on the entire constellation. The RMS of the positioning errors for the Galileo-only case are $0.43-1.20 \mathrm{~m}, 0.52-1.29 \mathrm{~m}$, and $1.34-3.25 \mathrm{~m}$ in the east component, north component, and up component, respectively, which are comparable to the results of the GPS-only case despite the smaller number of satellites and larger PDOPs. Galileo shows even better homogeneity and higher accuracy in the up component, which may be attributed to the more accurate $\mathrm{NeQuick}$ ionospheric model and the better precision of E1. By combining the horizontal and vertical RMSs, Galileo shows a three-dimensional (3D) positioning accuracy of $1.74-3.40 \mathrm{~m}$, while the 3D positioning accuracy of GPS is $1.99-4.5 \mathrm{~m}$. For the Galileo/GPS combined SPP, an initial weight ratio of 1:1 is appropriate for GPS and Galileo code observations. Compared with the GPS-only solution, the positioning accuracy of the Galileo/GPS

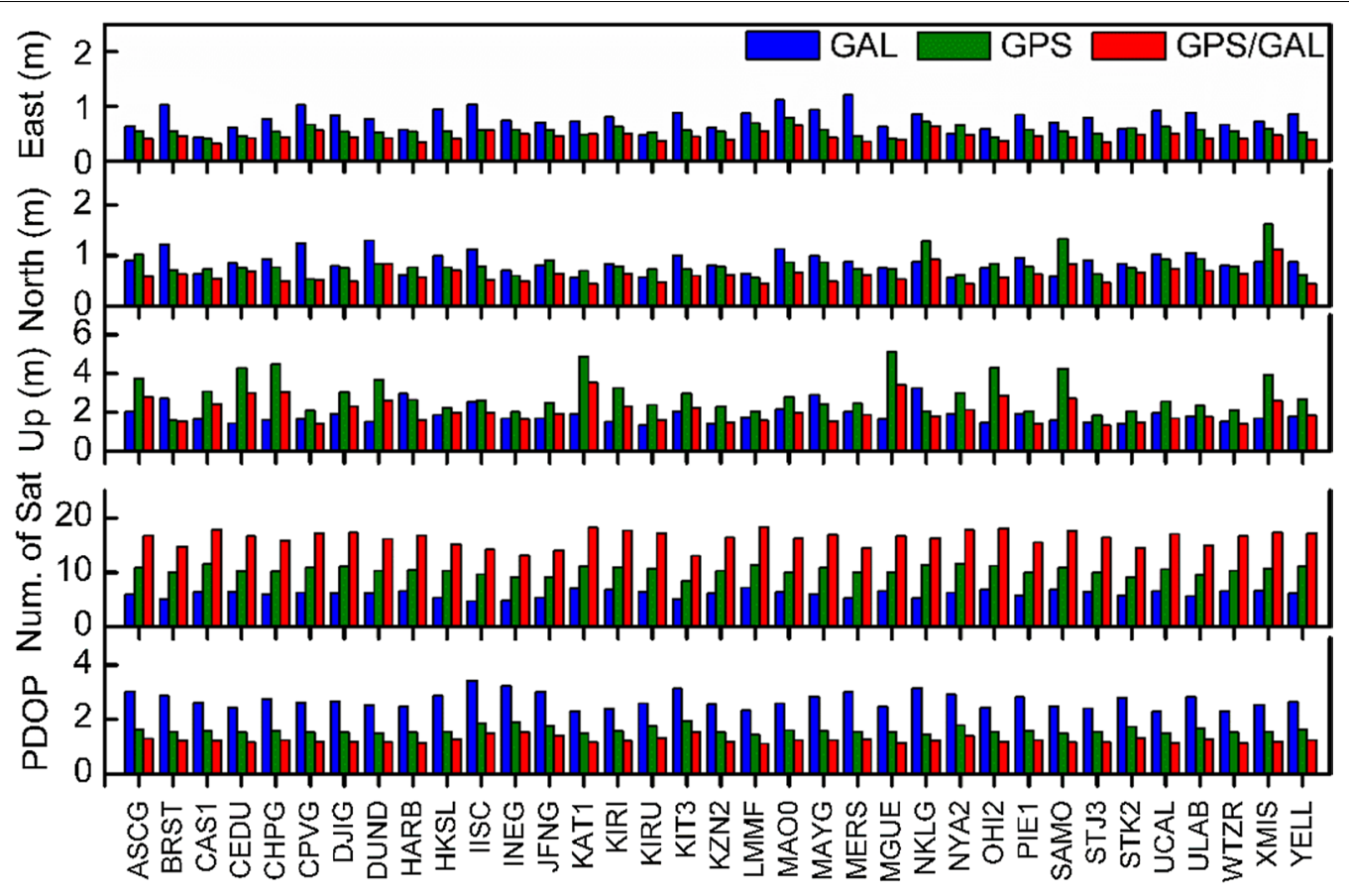

Station

Fig. 11 RMS statistics of positioning errors, average number of satellites and PDOP at 35 stations during DOY 200-206 of 2018 
solution is improved by $0.11 \mathrm{~m}, 0.20 \mathrm{~m}$, and $0.81 \mathrm{~m}$ in the east component, north component, and up component, respectively.

In addition to the E1 signal, positioning with E5, E5a, and E5b signals are also analyzed. Two representative stations, KZN2 and CUBS, equipped with the Trimble NETR9 and Septentrio PolaRx5 receiver are shown in Fig. 12. While the results exhibit similar accuracy for different frequencies, the RMS statistics can be ordered from low to high as E1, E5, and E5a/E5b. To confirm this finding, the quality of the signals was assessed from the perspectives of the carrier-to-noise density ratio (C/N0) and code multipath, as shown in Fig. 13. For KZN2, the C/N0 of E5 is significantly larger than that of other frequencies, and the multipath effect can be ordered from low to high as E5, E1, and E5a or E5b. For CUBS, the multipath effect of E1 is slightly larger relative to the E5a or E5b signals, which is also reported in Zaminpardaz and Teunissen [1]. Despite this finding, the error RMSs of E1 are slightly better than the other signals because the ionosphere delay of $\mathrm{E} 1$ is relatively smaller, i.e., the ionosphere delay of E5 is amplified by the factor $\frac{f_{\mathrm{E}}^{2}}{f_{\mathrm{E} 1}^{2}}=1.75$ compared with E1. Note that E5 shows the best signal quality and the measurement noise is significantly lower with respect to the other three signals, which can be attributed to its broad bandwidth and the advanced alternative binary offset carrier (AltBOC) modulation.

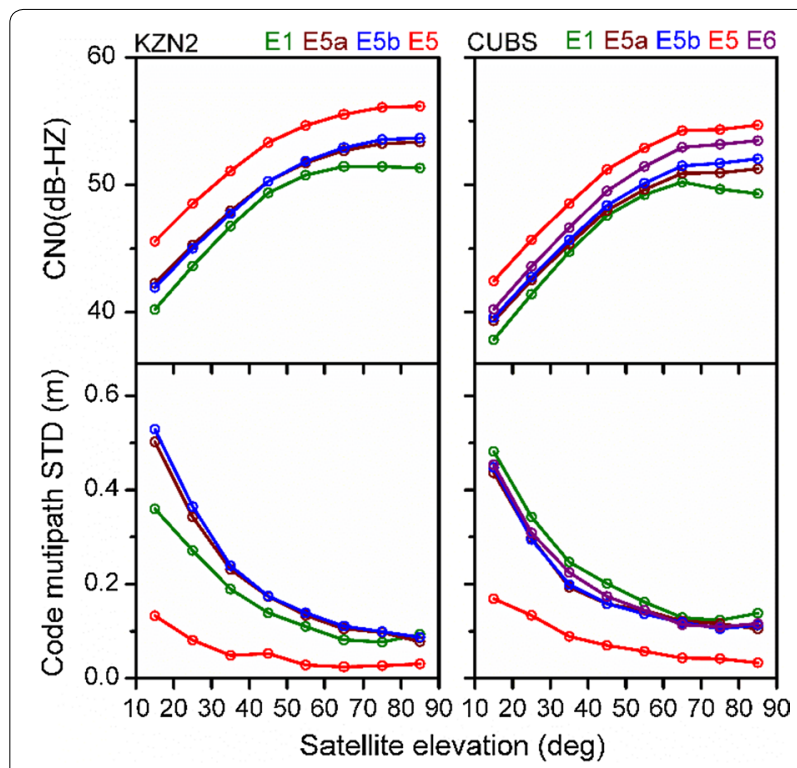

Fig. $13 \mathrm{C} / \mathrm{N} 0$ and Code multipath effect with different frequencies based on all Galileo observations for stations KZN2 (left) and CUBS (right) during DOY 200-206 of 2018

PPP results and analysis

Table 3 summarizes the annual RMS statistics of static and kinematic PPP daily solutions in the east (E), north $(\mathrm{N})$ and up $(\mathrm{U})$ directions, as well as their convergence

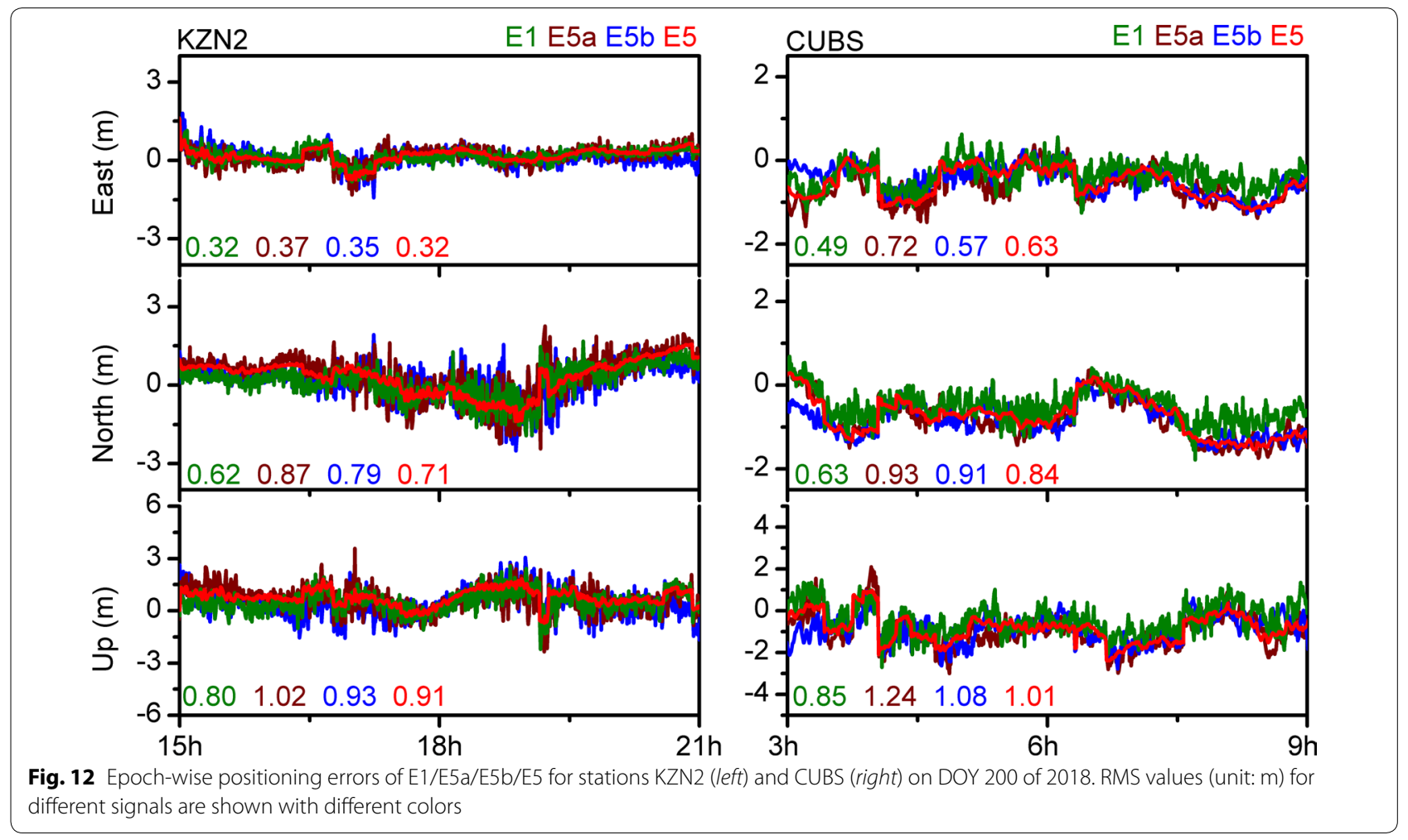


time in four cases. First, we admit that the positioning performance of the current Galileo is worse than that of the GPS due to the smaller number of visible satellites and the poorer geometry of Galileo, as shown in Fig. 11. With the exception of the entire constellation solutions in the 2016, 2017, and 2018a cases, case 2018b was conducted with the same number of satellites (i.e., 7) for the Galileo and GPS-only solutions. For static PPP, the RMS values of Galileo-only are $15.8 \mathrm{~mm}, 11.0 \mathrm{~mm}$, and $21.6 \mathrm{~mm}$ in the $\mathrm{E}$ direction, $\mathrm{N}$ direction, and $\mathrm{U}$ direction, respectively, in 2016. With the intensive launch of Galileo satellites in the past 2 years, more satellites are available with better geometry, and thus, the positioning accuracy has increased to $4-5 \mathrm{~mm}$ horizontally and $15 \mathrm{~mm}$ vertically since 2017 . The average convergence time of Galileo-only static PPP increased from $65.5 \mathrm{~min}$ in 2016 to $36.2 \mathrm{~min}$ in 2017. However, the accuracy and convergence time remain worse than that of GPS-only by a factor of 1.5.

For kinematic PPP, the horizontal RMS of Galileoonly decreased from 47.7 and $44.7 \mathrm{~mm}$ in 2016, to 31.6 and $24.2 \mathrm{~mm}$ in 2017. However, no significant improvement was attained for the vertical positioning accuracy, which was approximately $63.6 \mathrm{~mm}$ in the past few years. The average convergence time of Galileo-only kinematic PPP in 2018a is $87.3 \mathrm{~min}$, which is twice lower than that of GPS-only. By combining the Galileo and GPS observations, the positioning accuracy is significantly increased compared with that of Galileo-only, while it is comparable with that of GPS-only. Note that adding the Galileo observations can reduce the average convergence time by $32.9 \%$ and $50.2 \%$ for the GPS-only static solution and kinematic PPP solution, respectively. For 2018b, 3 h GPS
PPP solutions with 7 average visible satellites show comparable accuracy and average convergence time with respect to Galileo for the same number of satellites.

Figures 14 and 15 show the station-specific positioning results of the horizontal RMS and vertical RMS in 2018a. Similarly, the Galileo/GPS combined solution shows the best performance followed by the GPS-only solution, and the Galileo-only solution performs the worst. A uniform positioning accuracy can be globally achieved with the current Galileo system due to the global coverage.

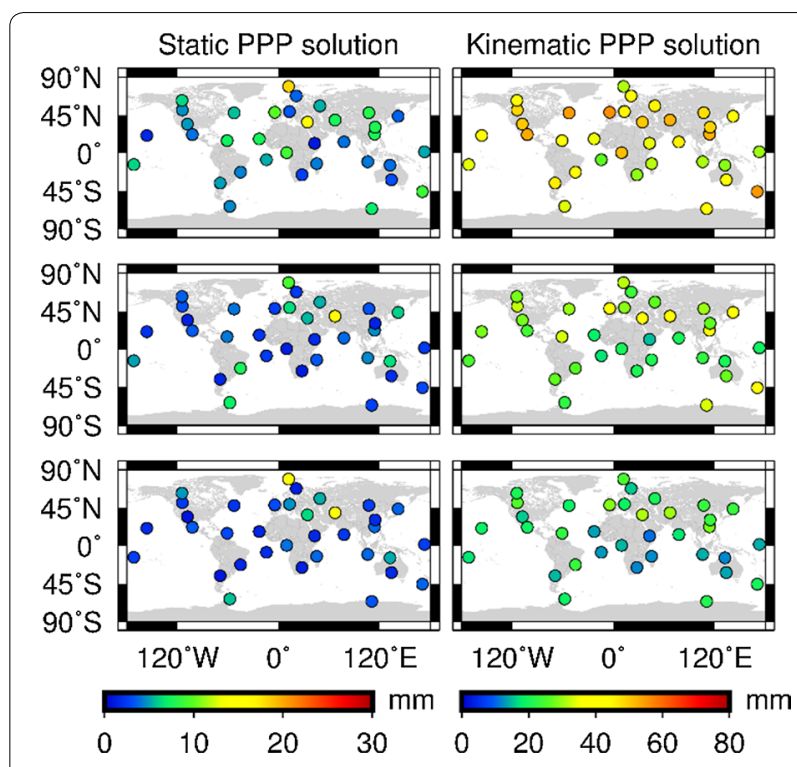

Fig. 14 Horizontal RMS of static PPP (left) and kinematic PPP (right) for Galileo-only (top), GPS-only (middle), and combined Galileo/GPS (bottom)

Table 3 Annual RMS statistics of the static PPP solution and kinematic PPP solution in East (E), North (N), and Up $(U)$ directions and the convergence time $(T)$ for a week in 2016, 2017, 2018a, and 2018b with the GPS and Galileo constellation, respectively

\begin{tabular}{|c|c|c|c|c|c|c|c|c|c|c|c|c|}
\hline \multirow[t]{2}{*}{ Mode } & \multicolumn{4}{|l|}{ GPS } & \multicolumn{4}{|c|}{ Galileo } & \multicolumn{4}{|c|}{ Combined GPS/GAL } \\
\hline & $E$ & $N$ & $U$ & $T$ & $E$ & $N$ & $U$ & $T$ & $E$ & $N$ & $U$ & $T$ \\
\hline \multicolumn{13}{|l|}{ Static } \\
\hline 2016 & 3.8 & 6.7 & 10.2 & 26.7 & 15.8 & 11.0 & 21.6 & 64.5 & 3.8 & 6.5 & 13.5 & 24.1 \\
\hline 2017 & 3.4 & 2.9 & 10.5 & 25.3 & 5.0 & 3.9 & 15.2 & 41.4 & 3.8 & 3.0 & 8.5 & 18.9 \\
\hline $2018 a$ & 2.8 & 2.9 & 8.3 & 25.5 & 4.5 & 3.7 & 14.3 & 36.2 & 2.4 & 2.7 & 6.7 & 17.1 \\
\hline $2018 b$ & 24.2 & 7.8 & 28.7 & 32.2 & 17.2 & 8.3 & 26.9 & 31.0 & - & - & - & - \\
\hline \multicolumn{13}{|c|}{ Kinematic } \\
\hline 2016 & 21.4 & 15.3 & 44.6 & 46.5 & 47.7 & 44.7 & 62.8 & 125.4 & 20.8 & 16.5 & 44.5 & 38.8 \\
\hline 2017 & 22.1 & 16.6 & 44.5 & 45.2 & 31.6 & 24.2 & 63.6 & 92.2 & 16.2 & 11.7 & 38.5 & 26.2 \\
\hline $2018 a$ & 22.0 & 16.0 & 45.1 & 44.6 & 32.4 & 24.4 & 63.4 & 87.3 & 15.4 & 11.9 & 38.5 & 22.2 \\
\hline $2018 b$ & 46.9 & 31.8 & 78.6 & 73.0 & 53.0 & 32.1 & 77.5 & 72.1 & - & - & - & - \\
\hline
\end{tabular}

2018a and $2018 \mathrm{~b}$ denote daily solutions based on the entire constellation and 3-h solutions based on 7 average visible satellites, respectively (unit: mm for ENU; min for $T$ ) 


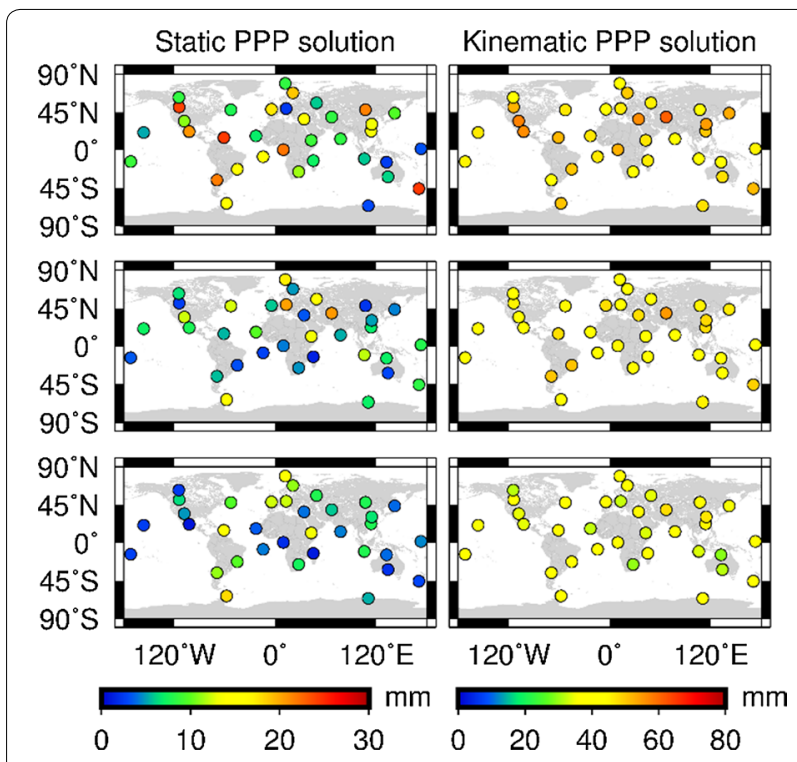

Fig. 15 Vertical RMS of static PPP (left) and kinematic PPP (right) for Galileo-only (top), GPS-only (middle), and combined Galileo/GPS (bottom)

The visible Galileo satellite number and PDOP of each station shown in Fig. 11 confirm this point of view. For static PPP, the RMSs of the Galileo-only solutions are within $10 \mathrm{~mm}$ horizontally, and the vertical RMSs is primarily less than $20 \mathrm{~mm}$. For kinematic PPP, the RMSs of Galileo-only solutions are predominantly within $40 \mathrm{~mm}$ horizontally and $60 \mathrm{~mm}$ vertically. Note that the statistics are different for stations located at different places, and there is no obvious correlation between such differences and the geographic location, especially regarding the statistics of the up component.

Although the aforementioned Galileo SISRE is smaller than that of GPS, the positioning accuracy of Galileo remains inferior to that of GPS. This finding is reasonable when we acknowledge the following two facts First, Galileo is in the phase of constellation deployment. Although 26 satellites are currently in orbit, 8 of these satellites are under commissioning or testing. For a specific station, only 6 or 7 Galileo satellites can be simultaneously observed, while the number of available satellites is 10-12 for GPS. As a result, the PDOP of Galileo is significantly larger than that of GPS. Second, due to limited knowledge about the new satellites and signals, orbit modeling of solar radiation pressure and phase center offset calibrations are not mature, i.e., the GPS L2 receiver PCOs and phase center variation (PCVs) were employed as an alternative in the Galileo PPP solution due to the lack of precise antenna phase center calibration for the Galileo E5a signal. These issues are expected to be addressed in the future.

\section{Conclusions}

Within this study, we presented an evolution analysis of SISRE and point positioning performance for the Galileo constellation from 2015 through 2018. The SISRE decreased from $0.58 \mathrm{~m}$ (2015) to $0.23 \mathrm{~m}$ (2018), which is better than the newest generation of the GPS (Block IIF). For FOC- 1 and 2 in the testing phase, the broadcast ephemeris shows a deficiency in elliptical orbit determination, which causes significantly large orbit errors when satellites pass the perigee, apogee, and intermediate point. The clocks of FOC- 1 and 2 have consistent accuracy compared with other operational satellites.

Positioning accuracy with the Galileo single-frequency E1 signal has achieved comparable (or even better) results with respect to the GPS L1 signal. The positioning RMS can be ordered from low to high as E1, E5, and E5a or E5b by comparing different Galileo signals. The smallest random noises of positioning error are observed in the solution with the E5 signal due to its excellent signal quality. In addition, static daily PPP solutions have a precision of approximately 4-7 $\mathrm{mm}$ in the horizontal component and 8-17 $\mathrm{mm}$ in the up component, and the precision is approximately $4 \mathrm{~cm}$ horizontally and $6 \mathrm{~cm}$ vertically for kinematic PPP. The average convergence times are $36.2 \mathrm{~min}$ and $87.3 \mathrm{~min}$ for the Galileo-only static PPP and Galileo-only kinematic PPP, respectively. Galileo can achieve a comparable accuracy and convergence time with respect to the GPS when the same number of visible satellites are employed. With the increasing number of operational satellites and further dedicated calibrations, such as the antenna phase center offset, more accurate results are expected for Galileo in the near future.

\section{Abbreviations}

SISRE: signal-in-space range error; FOC: full operational capability; SPP: single point positioning; PPP: precise point positioning; GNSS: global navigation satellite system; IOV: in-orbit validation; BDS: BeiDou navigation satellite system; GEO: geostationary Earth orbit; IGSO: inclined geosynchronous satellite orbit; MEO: medium Earth orbit; RMS: root mean square; MGEX: multi-GNSS experiment; IGS: international GNSS service; DCB: differential code bias; TGD: time group delay; RAFS: rubidium atomic frequency standards.

\section{Acknowledgements}

The contribution of data from IGS MGEX is appreciated.

\section{Authors' contributions}

FG conceived and designed the experiments. WW performed the experiments, analyzed the data and wrote the paper. FG and JZ reviewed the paper. All authors read and approved the final manuscript.

\section{Funding}

This research was funded by the National Natural Science Foundation of China (No. 41774034), and the National Key Research and Development Program of China (No. 2016YFB0501803, No. 2017YFB0503402). 


\section{Availability of data and materials}

All data in this paper are online at ftp://cddis.gsfc.nasa.gov or ftp://igs.ign.fr/ pub/igs/products/mgex/.

\section{Ethics approval and consent to participate}

Not applicable.

\section{Consent for publication}

Not applicable.

\section{Competing interests}

The authors declare that they have no competing interests.

\section{Author details}

1 School of Geodesy and Geomatics, Wuhan University, 129 Luoyu Road, Wuhan 430079, China. ${ }^{2}$ College of Civil Engineering, Nanjing Forestry University, 159 Lonpan Road, Nanjing 210037, China.

Received: 1 August 2019 Accepted: 19 December 2019

Published online: 03 February 2020

\section{References}

1. Zaminpardaz, S., \& Teunissen, P. J. G. (2017). Analysis of Galileo IOV + FOC signals and E5 RTK performance. GPS Solutions, 21(4), 1855-1870. https:// doi.org/10.1007/s10291-017-0659-9.

2. Montenbruck, O., Steigenberger, P., \& Hauschild, A. (2015). Broadcast versus precise ephemerides: a multi-GNSS perspective. GPS Solutions, 19(2), 321-333. https://doi.org/10.1007/s10291-014-0390-8.

3. Heng, L., Gao, G. X., Walter, T., \& Enge, P. (2011). Statistical characterization of GPS signal-in-space errors. In Proceedings of the ION ITM 2011, San Diego, California, USA, January 24-26, pp. 312-319.

4. Heng, L., Gao, G. X., Walter, T. F., \& Enge, P. K. (2012). GPS signal-in-space integrity performance evolution in the last decade: data mining $400,000,000$ navigation messages from a global network of 400 receivers. IEEE Transactions on Aerospace and Electronic Systems, 48(4), 2932-2946. https://doi.org/10.1109/TAES.2012.6324670.

5. Warren, D. L. M., \& Raquet, J. F. (2003). Broadcast vs precise GPS ephemerides: A historical perspective. GPS Solutions, 7(3), 151-156. https://doi. org/10.1007/s10291-003-0065-3.

6. Chen, L., Jiao, W., Huang, X., Geng, C., Ai, L., Lu, L., \& Hu, Z. (2013). Study on signal-in-space errors calculation method and statistical characterization of BeiDou navigation satellite system. In Proceedings of China satellite navigation conference (CSNC). Lecture notes in electrical engineering 243 (pp. 423-434). Berlin: Springer. https://doi.org/10.1007/978-3-642-37398 $-5 \_39$.

7. Wu, J., Wu, S., Hajj, G., Bertiger, W., \& Lichten, S. (1993). Effects of antenna orientation on GPS carrier phase. Manuscr Geod, 18, 91-98.

8. Lucas Rodriguez, R. (2013). Galileo IOV status and results. In Proceedings of ION GNSS + 2013, Nashville, Tennessee, USA, September 16-20, pp. 3065-3093
9. Perea, S., Meurer, M., Rippl, M., Belabbas, B., \& Joerger, M. (2017). URA/SISA analysis for GPS and Galileo to support ARAIM. Navigation, 64(2), 237-254. https://doi.org/10.1002/navi.199.

10. Montenbruck, O., Steigenberger, P., \& Hauschild, A. (2018). Multi-GNSS signal-in-space range error assessment-methodology and results. Advances in Space Research, 61(12), 3020-3038. https://doi.org/10.1016/j. asr.2018.03.041.

11. Cao, W., Hauschild, A., Steigenberger, P., Langley, R. B., Urquhart, L., Santos, M., \& Montenbruck, O. (2010). Performance evaluation of integrated GPS/ GIOVE precise point positioning. In Proceedings of the ION ITM 2010, San Diego, California, USA, January 25-27, pp. 540-552.

12. Gioia, C., Borio, D., Angrisano, A., Gaglione, S., \& Fortuny-Guasch, J. (2015). A Galileo IOV assessment: measurement and position domain. GPS Solutions, 19(2), 187-199. https://doi.org/10.1007/s10291-014-0379-3.

13. Guo, F., Li, X., Zhang, X., \& Wang, J. (2017). The contribution of multi-GNSS experiment (MGEX) to precise point positioning. Advances in Space Research, 59(11), 2714-2725. https://doi.org/10.1016/j.asr.2016.05.018.

14. Guo, F., Li, X., Zhang, X., \& Wang, J. (2017). Assessment of precise orbit and clock products for Galileo, BeiDou, and QZSS from IGS multi-GNSS experiment (MGEX). GPS Solutions, 21(1), 279-290. https://doi.org/10.1007/ s10291-016-0523-3.

15. Steigenberger, P., \& Montenbruck, O. (2017). Galileo status: orbits, clocks, and positioning. GPS Solutions, 21(2), 319-331. https://doi.org/10.1007/ s10291-016-0566-5

16. Paziewski, J., Sieradzki, R., \& Wielgosz, P. (2018). On the applicability of Galileo FOC satellites with incorrect highly eccentric orbits: an evaluation of instantaneous medium-range positioning. Remote Sensing, 10(2), 208. https://doi.org/10.3390/rs10020208.

17. Dow, J. M., Neilan, R., \& Rizos, C. (2009). The international GNSS service in a changing landscape of global navigation satellite systems. Journal of Geodesy, 83(3-4), 191-198. https://doi.org/10.1007/s00190-008-0300-3.

18. Kouba, J., \& Heroux, P. (2001). Precise point positioning using IGS orbit and clock products. GPS Solutions, 5(2), 12-28. https://doi.org/10.1007/ pl00012883.

19. GSA. (2017). Galileo satellite metadata. https://www.gsc-europa.eu/ support-to-developers/galileo-iov-satellite-metadata. Accessed November 28, 2017.

20. European Union. (2016). European GNSS (Galileo) open service signal in space interface control document. OS SIS ICD, Issue 1.3, December 2016.

21. Boehm, J., Heinkelmann, R., \& Schuh, H. (2007). Short note: a global mode of pressure and temperature for geodetic applications. Journal of Geodesy, 81(10), 679-683. https://doi.org/10.1007/s00190-007-0135-3.

22. Quiles, A. (2017). Galileo system status update. In Proceedings fo ION GNSS + 2017, Portland, Oregon, USA, September 25-29, pp. 865-886.

23. Blonski, D. (2015). Galileo system status. In Proceedings of the ION GNSS + 2015, Tampa, Florida, USA, September 14-18, pp. 1241-1263.

\section{Publisher's Note}

Springer Nature remains neutral with regard to jurisdictional claims in published maps and institutional affiliations.

\section{Submit your manuscript to a SpringerOpen ${ }^{\odot}$ journal and benefit from:}

- Convenient online submission

- Rigorous peer review

- Open access: articles freely available online

- High visibility within the field

- Retaining the copyright to your article

Submit your next manuscript at $\boldsymbol{\nabla}$ springeropen.com 